\title{
Quantized conductance in an AlAs 2D electron system quantum point contact
}

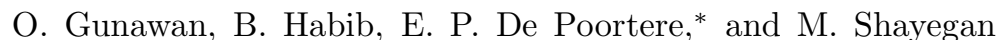 \\ Department of Electrical Engineering, Princeton University, Princeton, New Jersey 08544
}

(Dated: September 12, 2018)

\begin{abstract}
We report experimental results on a quantum point contact (QPC) device formed in a wide AlAs quantum well where the two-dimensional electrons occupy two in-plane valleys with elliptical Fermi contours. To probe the closely-spaced, one-dimensional electric subbands, we fabricated a point contact device defined by shallow-etching and a top gate that covers the entire device. The conductance versus top gate bias trace shows a series of weak plateaus at integer multiples of $2 e^{2} / h$, indicating a broken valley degeneracy in the QPC and implying the potential use of QPC as a simple "valley filter" device. A model is presented to describe the quantized energy levels and the role of the in-plane valleys in the transport. We also observe a well-developed conductance plateau near $0.7 \times 2 e^{2} / h$ which may reflect the strong electron-electron interaction in the system.

PACS numbers: 73.23.-b, 73.23.Ad, 73.61.Ey
\end{abstract}

\section{INTRODUCTION}

The quantum point contact (QPC) is a prime example of a mesoscopic structure that demonstrates a striking quantum mechanical ballistic transport phenomenon. It exhibits a quantization of conductance in units of $G_{0}=2 e^{2} / h$ where the factor of 2 accounts for spin degeneracy 1.2 This effect has been observed in many twodimensional electron systems (2DESs) with mean-freepath longer than the QPC channel length. It arises as a consequence of the quantization of transverse momentum and full transmission of the one-dimensional modes in the constriction, reminiscent of quantum Hall effect where the absence of backscattering leads to quantized plateaus in the Hall resistance. Quantized conductance in QPCs has been observed and studied in many 2D carrier systems including electrons in $\mathrm{GaAs}, \stackrel{1.2}{\stackrel{2}{2}} \mathrm{SiGe}, \stackrel{3.4}{=} \mathrm{GaN}, \underline{5}$ $\mathrm{InSb}, \underline{6}$ and holes in $\mathrm{GaAs}^{7.8}$

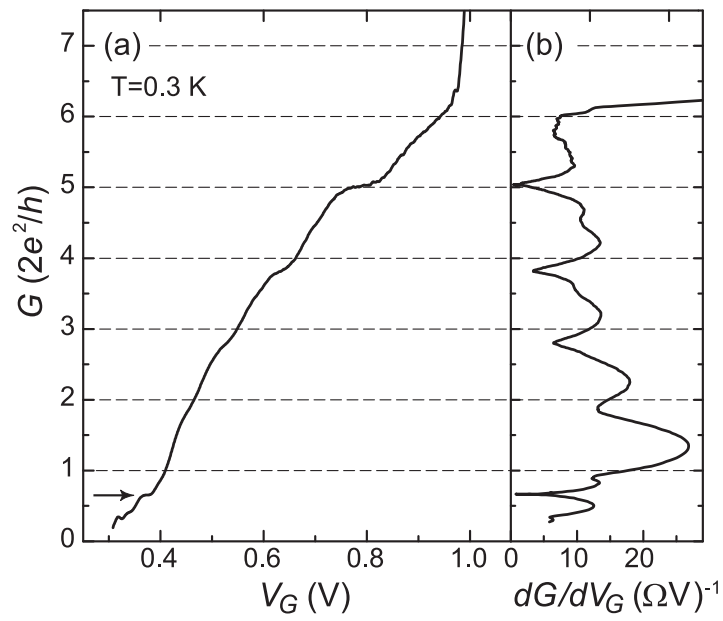

FIG. 1: (a) Conductance vs gate voltage. The arrow indicates the "0.7 structure". (b) The transconductance $d G / d V_{G}$. The minima, nearly periodic in $2 e^{2} / h$ (except for the " 0.7 structure"), indicate developing plateaus in the conductance trace.
Here we report transport measurements of a QPC device realized in a 2DES confined to an $11 \mathrm{~nm}$-wide, modulation-doped AlAs quantum well grown on a GaAs (001) substrate. The novelty of this system is that the 2D electrons occupy conduction band valleys located at the $\mathrm{X}$ points of the Brillouin zone. There are two anisotropic, elliptical Fermi contours, each characterized by a heavy longitudinal mass $m_{l}=1.1 m_{0}$ and a light transverse mass $m_{t}=0.2 m_{0}$, where $m_{0}$ is the electron mass in vacuum. ${ }^{9}$ Our QPC device is fabricated via shallow-etching and depositing a top gate that covers the entire sample and controls the density in both the $2 \mathrm{D}$ reservoir and the QPC channel. As outlined in the abstract, the conductance vs gate bias trace of this device exhibits several interesting features, some of which are puzzling. In the next section, we summarize these observations and briefly describe their origin; the rest of the paper is devoted to the details of the device and a quantitative explanation of conductance vs gate bias data.

\section{QUANTIZED CONDUCTANCE}

Figure 1 presents our main finding. It shows the conductance $(G)$ trace of the QPC device measured at $T=0.3$ $\mathrm{K}$ as a function of the applied gate voltage $\left(V_{G}\right)$ and the corresponding transconductance trace $\left(d G / d V_{G}\right)$ that accentuates the features in $G$. Three features of the data are noteworthy. First, we observe developing quantized conductance steps near integer multiples $(N)$ of $2 e^{2} / h$ up to $N=5$; these steps are exhibited more clearly in the transconductance plot of Fig. 团b) ${ }^{10}$ Second, the $2 e^{2} / h$ conductance plateaus seem to get stronger monotonically at higher $N$. Third, we observe a particularly strong plateau near $0.7 \times 2 e^{2} / h$.

Perhaps the most puzzling feature of Fig. 1 data is that, even though the system is expected to have a twofold valley degeneracy, we do not observe quantized steps at integer multiples of $4 e^{2} / h$ but instead at multiples of $2 e^{2} / h$. As we discuss in detail in the remainder of the paper, this happens because the mass anisotropy breaks 
the valley degeneracy of the QPC subband energy levels. The valley with higher mass in the QPC (lateral) confinement direction has lower energy and therefore dominates the low-lying subband states. Moreover a small but finite residual valley splitting present in the system further helps lift the valley degeneracy.

Next, the overall weakness of the observed plateaus in Fig. 1 imply that the QPC energy subband spacings are comparable to $k_{B} T$, where $k_{B}$ is the Boltzmann constant and $T$ is the system temperature. This is not surprising, considering the rather large effective mass of electrons (compared to, e.g., GaAs 2D electrons) which leads to small subband spacings. The fact that the conductance plateaus get stronger monotonically at higher conductance steps [Fig. [1 (b)], on the other hand, is somewhat puzzling. This distinct feature is not observed in conventional surface split-gate QPCs ${ }^{1.2}$. We attribute it mainly to two characteristics of our device. First, since our QPC constriction is defined using shallow-etching and gating (in contrast to only gating using split-gate), we expect the lateral confinement potential to be strong 11.12 resembling a square well potential (Fig. 21). In such a potential, the energy subband spacing $\Delta E_{N+1, N}$ increases at higher $N$, therefore at a given temperature, the subband spacing will be better resolved. Second, the electron mean-free-path in our device is comparable to the length of the channel. As we increase the gate voltage, the density in the QPC channel increases, resulting in higher electron mobility and longer electron mean-free-path. $\underline{13}^{13}$ This results in a better transmission across the QPC and thus a better plateau formation at higher $V_{G}$. We note that, a monotonic increase in strength of $2 e^{2} / h$ plateaus suggests that the quantized energy levels originate from a single valley. Any accidental degeneracy between $X$ and $Y$ valley $1 \mathrm{D}$ energy levels in the $\mathrm{QPC}$ would lead to non-monotonic energy level spacings and would cause a deterioration or missing of conductance plateaus, in contrast to the monotonic increase in plateau strength as observed here.

Finally, the conductance plateau we observe near $0.7 \times 2 e^{2} / h$ is particularly strong compared to the other plateaus in our QPC device or the "0.7 structure" plateaus reported for QPCs fabricated using other 2D systems. The origin of the " 0.7 structure" is still unknown although there is a general consensus that, unlike the integer plateaus, it may be caused by electronelectron interaction 12.14.15.16.17.18 Our observation is consistent with such interpretation as we indeed do expect interaction to be strong in our system because of the heavy electron effective mass.

\section{DEVICE STRUCTURE}

We used a modulation-doped AlAs quantum well (QW) grown by molecular beam epitaxy on a (001) GaAs substrate. The well is $11 \mathrm{~nm}$ wide, resides $\simeq 110 \mathrm{~nm}$ below the surface and is flanked by undoped and $\mathrm{Si} \delta$-doped

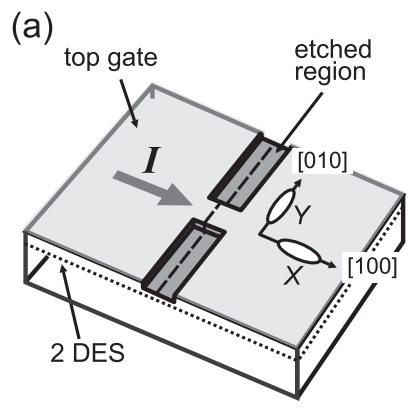

(b)

(c)

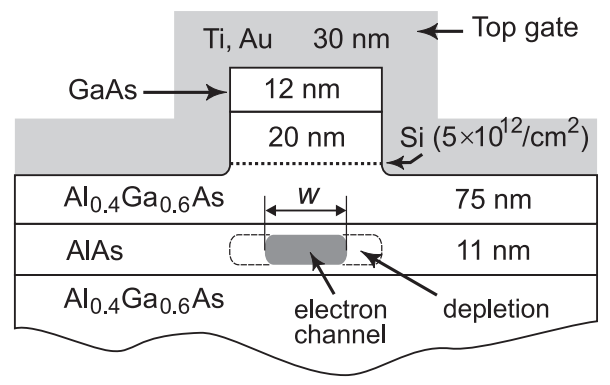

FIG. 2: (a) Device schematic of the shallow-etched QPC. The $k$-space orientation of the in-plane valleys labeled as $X$ and $Y$ is also shown. (b) Confinement potential along the dashed-line in (a). The Fermi energy varies with the gate bias voltage. (c) Device cross-section along the dashed line in (a).

layers of $\mathrm{Al}_{0.4} \mathrm{Ga}_{0.6} \mathrm{As}$ [Fig. 2( $\left.\left.\mathrm{c}\right)\right] \stackrel{13}{\underline{13}}$ The QPC is fabricated on a standard Hall bar device, defined by optical lithography.

In most QPC devices, the channel is defined by a pair of surface metal split gates to control the channel's electrical width using an applied voltage bias to the gates $\frac{1.2}{.6}$ In such a device the width of the confinement potential changes considerably while the Fermi level $\left(E_{F}\right)$ in the 2DES reservoir remains constant. In a system with large electron effective mass such as in AlAs 2DES, the energy level spacings are expected to be very small, rendering the observation of quantized conductance challenging. We tried a number of split-gate structures with no success in observing quantized conductance steps. Experiments on high quality AlAs quantum wires fabricated via the cleaved-edge-overgrowth technique, have not yielded clear quantized plateaus either ${ }^{19}$ We therefore took an alternative approach and defined the QPC constriction by shallow-etching to introduce a strong QPC confinement, $, 11,12$ and then covered the entire device by a $\mathrm{Ti} / \mathrm{Au}$ top gate that controls the $2 \mathrm{DES}$ density (and thus $E_{F}$ ) in the reservoir and in the QPC constriction [Fig. 2(a)]. As we demonstrate later in the paper, this device structure allows us to directly probe the energy spacing between the QPC quantized energy levels. One disadvantage is that the background resistance, from the series resistance of the 2DES reservoir regions flanking the point contact, varies with the gate voltage; however, this background correction is found to be rather 
negligible as shown in Fig. 10 ${ }^{10}$

Our QPC constriction was defined by electron beam lithography to be $300 \mathrm{~nm}$ wide and $500 \mathrm{~nm}$ long with the transport direction aligned along [100] as shown in Fig. 2(a). Due to carrier depletion near the boundary of the constriction, however, the electrical channel width $(w)$ is expected to be smaller. We performed wetetching using $\mathrm{H}_{2} \mathrm{SO}_{4}: \mathrm{H}_{2} \mathrm{O}_{2}: \mathrm{H}_{2} \mathrm{O}$ (1:8:160) solution to a depth of $65 \mathrm{~nm}$ to remove the $\mathrm{Si}$ dopant layer, thus completely depleting the electrons under the etched region [Fig. 2(c)]. Using illumination and top/back gate biasing ${ }^{20}$ we were able to vary the 2 DES density $n_{2 D}$ between 2.4 to $4.5 \times 10^{15} / \mathrm{m}^{2}$ with a low temperature mobility of $\sim 15 \mathrm{~m}^{2} / \mathrm{Vs}$, implying a transport mean-freepath of $\sim 1 \mu \mathrm{m}$. The measurements were done in a ${ }^{3} \mathrm{He}$ cryostat system with $0.3 \mathrm{~K}$ base temperature and using standard phase sensitive lock-in technique in a four-wire configuration.

\section{ANALYSIS AND DISCUSSION}

To gain insight into the transport in the QPC and especially to understand the role of the two in-plane valleys, we present a simple model to describe the QPC energy levels by taking into account the QPC geometry and the electron system parameters. Our goal is to accurately explain the values of $V_{G}$ at which we observe the conductance plateaus. We start with an infinite square well model and a constant QPC channel width. However, as we will show, this description is unsatisfactory for our system, and thus later on we refine the model by treating the channel width as a gate voltage-dependent variable. We also corroborate this analysis with the channel width deduced from our low-field magnetoresistance data.

\section{A. Quantum point contact model}

Figures 3(a) and (b) present a model for the potential landscape around the QPC. In our device, as we increase the gate voltage, $E_{F}$ increases and crosses the quantized energy levels in the QPC, leading to quantized conductance steps. The Fermi energy can be obtained from the density $\left(n_{2 D}\right)$ in the $2 \mathrm{D}$ reservoirs on the two sides of the QPC. This density can be determined from Shubnikovde Haas oscillations and Hall resistance measurements as will be detailed in the next section (Figs. 团and 5 ). We start with the simple assumption that there are two inplane valleys $X$ and $Y$ with equal population in the 2D reservoirs participating in transport; $X$ and $Y$ refer to valleys with their major axes along [100] and [010] directions (see Figs. 2] and [3). Given $n_{2 D}$, we can calculate the Fermi energy $E_{F}=n_{2 D} / \rho$ where $\rho=2 m^{*} / \pi \hbar^{2}$ is the spin- and valley-degenerate $2 \mathrm{D}$ density of states; we used $m^{*}=\sqrt{m_{l} m_{t}}$. This $E_{F}$ is plotted as a function of $V_{G}$ in Fig. 3(c).
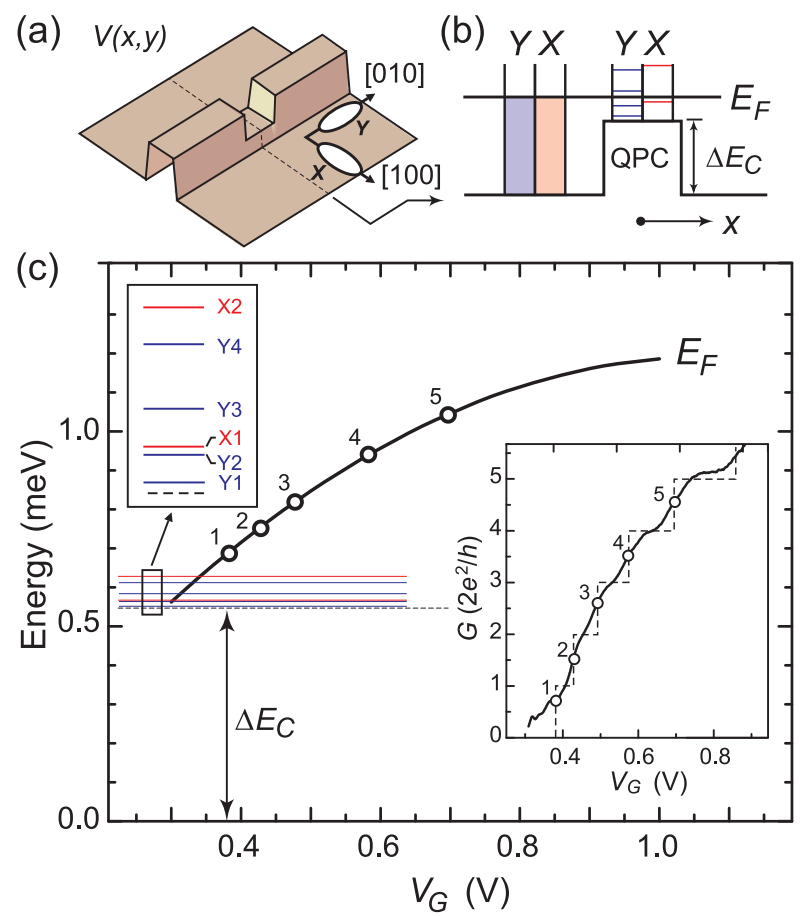

FIG. 3: (a) Schematic drawing showing the potential landscape surrounding the QPC and the orientations of the two in-plane valleys $X$ and $Y$. (b) Potential cross-section along the transport direction [dotted line in (a)]. (c) Dependence of the Fermi energy on to the gate voltage. The circles are the expected level crossings. Right inset: The conductance vs $V_{G}$ trace together with an idealized conductance step model to determine the level crossings (see text). Left inset: The QPC energy levels assuming a fixed channel width of $w=300 \mathrm{~nm}$. It is apparent that the energy levels do not fit the level crossings.

From the conductance trace in Fig. 1 we can estimate the gate voltages where $E_{F}$ crosses the $1 \mathrm{D}$ subbands in the QPC. Since the plateaus are not sharply defined in our experiment we cannot pinpoint exactly where these crossing points are. However the kinks at every $2 e^{2} / h$ in the conductance trace are clear. These kinks occur when $E_{F}$ lies in the middle of an energy gap. Thus the level crossings occur approximately at gate voltages half-way in between successive gate voltages where the kinks appear. Using this approximation we construct an idealized conductance steps trace shown by dashed lines in the inset of Fig. [3(c). The positions where the level crossings occur are marked as circles (labeled with their subband indices $N=1$ to 5 ).

Figure 3(b) assumes that the QPC has an elevated bottom potential, with an offset $\Delta E_{C}$, with respect to the 2DES reservoir potential. This is to model the fact that the QPC has a pinch-off voltage $V_{P}=0.3 \mathrm{~V}$ (Fig. (1) even though the density in the $2 \mathrm{D}$ reservoir is non-zero at this voltage. We take $\Delta E_{C}$ to be equal to $E_{F}$ at $V_{G}=V_{P}$.

In the QPC channel, the $X$ and $Y$ valleys have a differ- 


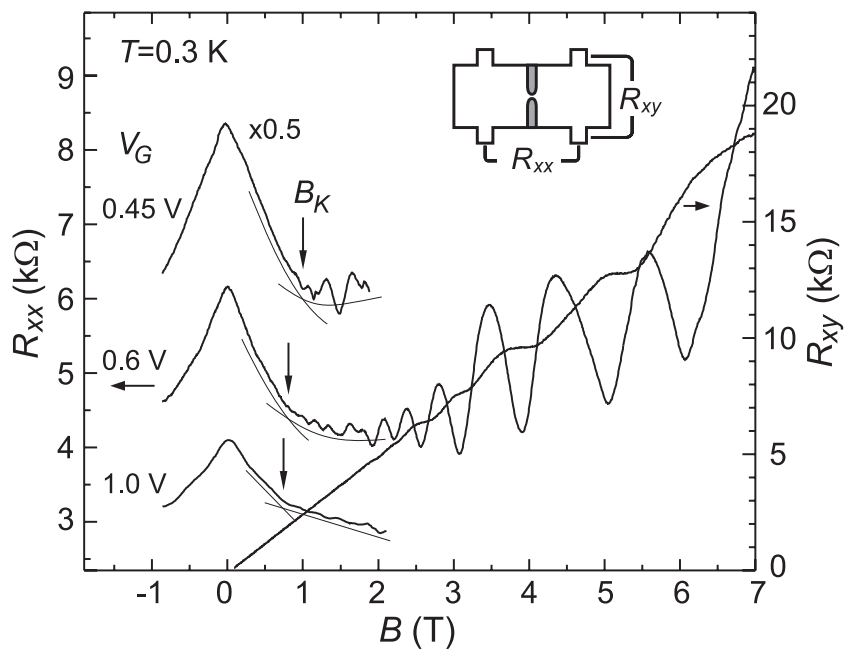

FIG. 4: Magnetoresistance traces for the QPC device. Inset shows the contact geometry for these traces. The kink in the $R_{x x}$ magnetoresistance trace, (marked by the arrow $B_{K}$ ), signals the field where the cyclotron orbit fits into the width of the QPC channel. For $V_{G}=0.6 \mathrm{~V}$, a Hall resistance $\left(R_{x y}\right)$ trace is also shown.

ent set of quantized energy levels as shown in Figure 3(b). This happens because, thanks to the different valley orientations, the electrons in the $X$ and $Y$ valleys have different effective masses along the lateral confinement direction. This mass anisotropy breaks the degeneracy of the quantized levels in the channel. Since the confinement potential is strong and quantized energies are small due to heavy electron effective mass, we can estimate the $1 \mathrm{D}$ subbands' energy levels using an infinite square well model: $E_{N}=N^{2} \hbar^{2} \pi^{2} / 2 m_{y}^{*} w^{2}$ where $m_{y}^{*}$ is the electron mass along the $\mathrm{QPC}$ confinement direction; $m_{y}^{*}$ is equal to $m_{l}\left(m_{t}\right)$ for the $Y(X)$ valley. Thus the ground state energy for valley $Y$ is lower than that of $X$ by a factor of $m_{l} / m_{t}=5.5$. Finally, given the channel width of $w=300 \mathrm{~nm}$, we calculated the first few subband levels for both $X$ and $Y$ valleys and plot them in Fig. 3(c). It is apparent that the energy levels do not agree with the expected level crossings. This points to the inadequacy of our simple model and demands further refinement, which we describe in the next section.

\section{B. Determination of QPC channel density and width}

Since the constriction is defined by etching, the removal of the dopant layer, together with the Fermi level pinning effect at the exposed surface, induces depletion regions that narrow the QPC channel. Simple theoretical estimates, confirmed by experimental results, indicate that the depletion width decreases with increasing 2DES density. ${ }^{21.22}$ In addition, as seen in Fig. 2( (c), the top gate covering the shallow-etched region comes to close proximity of the QPC channel (somewhat similar to a split-gate device) so that changing the gate bias may also influence

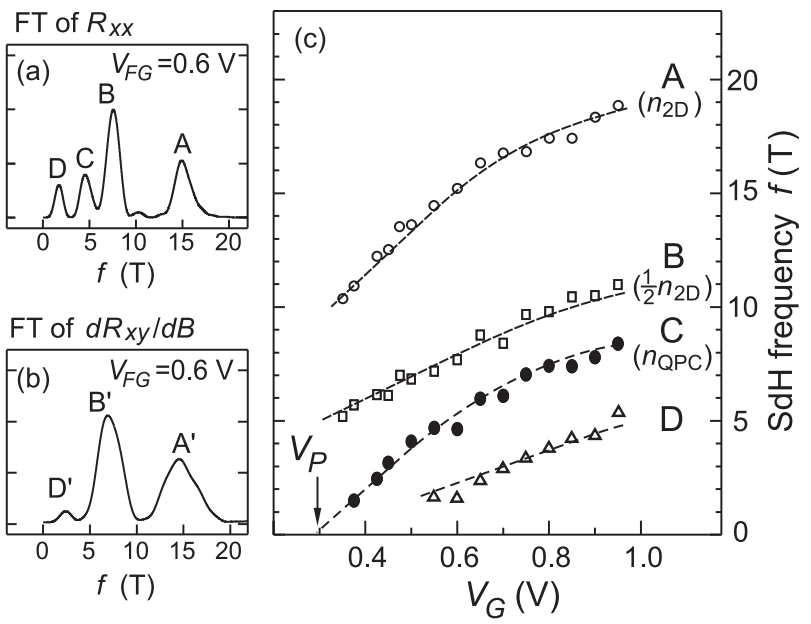

FIG. 5: (a) Fourier transform (FT) spectrum of $R_{x x}$ representing density components in the $2 \mathrm{D}$ reservoir and the QPC. (b) Fourier transform spectrum of $d R_{x y} / d B$ representing density components in the 2D reservoir alone. (c) Summary of the Fourier spectra peaks vs $V_{G}$.

the channel width electrostatically. Therefore, we expect that the channel width $w$ would increase at higher $V_{G}$ where the density is higher.

It is possible to estimate the channel width from the QPC magnetoresistance (MR) 23 Figure 4 shows the longitudinal MR $\left(R_{x x}\right)$ traces across the QPC and the transverse MR $\left(R_{x y}\right)$ measured in one of the reservoir regions (see Fig. 4 inset). The $R_{x x}$ traces exhibit strong negative MR at low field. This negative MR arises from the suppression of the constriction resistance by the magnetic field in the ballistic regime. As the magnetic field is increased, the electron backscattering rate is reduced and a larger fraction of the edge states are transmitted through the channel, resulting in a smaller resistance ${ }^{23}$ This behavior persists up to a magnetic field $B_{K}$ at which the classical cyclotron diameter equals the channel width. At $B_{K}$ there is a marked change in the MR slope, appearing as a "kink" in the trace (Fig. (4) that can be used to estimate the width of the QPC:

$$
w=2 \hbar k_{F} / e B_{K}
$$

where $k_{F}$ is the Fermi wavevector perpendicular to the width of the QPC 23

In our QPC there are two possible in-plane valleys, each with an elliptical cyclotron orbit, that could participate in the transport. For the $X$ and $Y$ valleys, the Fermi wavevectors along the transport direction (perpendicular to the channel width) can be written as: 24

$$
\begin{aligned}
k_{F, X}^{2} & =2 \pi n_{Q P C, X} \sqrt{m_{l} / m_{t}} \\
k_{F, Y}^{2} & =2 \pi n_{Q P C, Y} \sqrt{m_{t} / m_{l}}
\end{aligned}
$$

where $n_{Q P C, X}$ and $n_{Q P C, Y}$ are the $2 \mathrm{D}$ valley densities in the QPC for the $X$ and $Y$ valleys, respectively. These electron densities in the QPC channel can be determined 


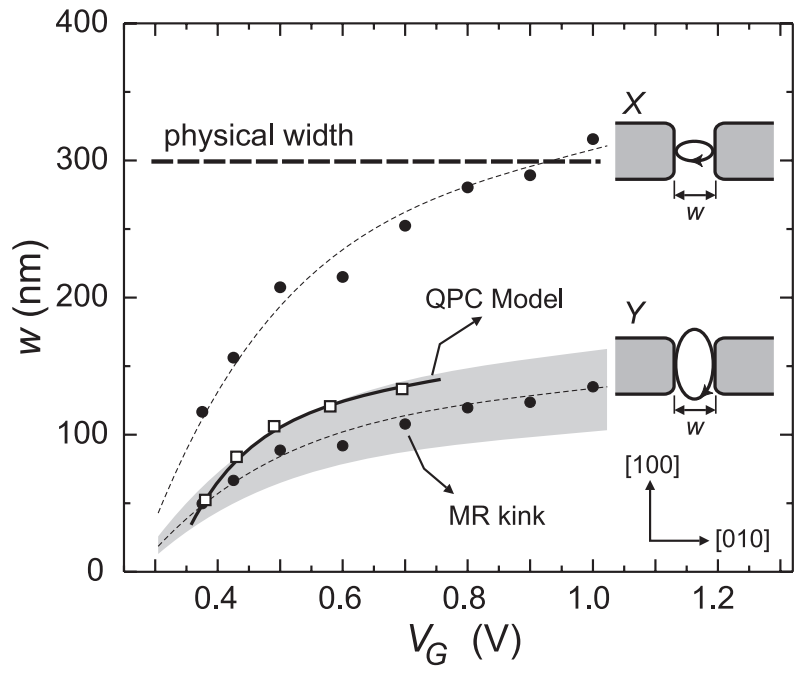

FIG. 6: QPC channel electrical width $(w)$, deduced from the kink in the magnetoresistance, for two possible cases where either valley $X$ or $Y$ is occupied; these cases are schematically illustrated on the right. Gray area denotes the error band for $w$ from the MR kink data. The open squares and solid line indicate $w$ determined from the QPC model (see text).

from the Fourier spectra of the Shubnikov-de Haas (SdH) oscillations as follows.

Since $R_{x x}$ is measured across the QPC (Fig. 4), it contains $\mathrm{SdH}$ oscillation contributions from both the $2 \mathrm{D}$ reservoirs and the QPC region ${ }^{25}$ Ideally, we could obtain density information in the $2 \mathrm{D}$ reservoirs from $\mathrm{MR}$ measurements in a plain $2 \mathrm{D}$ region adjacent to the $\mathrm{QPC}$. Unfortunately, there are no contacts available to measure such a region in our device. Thus, to obtain the density in the $2 \mathrm{D}$ reservoirs alone, we deduce the $\mathrm{SdH}$ oscillations from $d R_{x y} / d B$ that represents the "longitudinal MR" following the empirical resistance rule ${ }^{26}$ It has been shown that the Hall resistance is unaffected by the presence of the QPC constriction ${ }^{23}$

The Fourier spectra of $R_{x x}$ and $d R_{x y} / d B$ are shown in Figs. 囵(a) and (b). We observe three common frequency components labeled as A, B and D in Fig. 5 (a) [A', B', and D' in Fig. [5) (b) in the Fourier spectra of $R_{x x}$ and $d R_{x y} / d B$ traces. These frequency components are associated with total density (A) and half total density (B) in the $2 \mathrm{D}$ reservoir region ${ }^{27}$ (We will shortly return to peak $\mathrm{D}$ in the next section). We assign the frequency component $\mathrm{C}$, which appears only in $R_{x x}$ Fourier spectrum [Fig. [5)], to the total density in the QPC channel. Our justification for this assignment is two-fold. First, the frequency component $\mathrm{C}$ does not appear in the $\mathrm{SdH}$ spectrum of $d R_{x y} / d B$ that represents $\mathrm{SdH}$ oscillations from the $2 \mathrm{D}$ reservoir alone. Second, this frequency component extrapolates to zero at $V_{G}=0.3 \mathrm{~V}$ [Fig. 囵(c)] marking the pinch-off voltage $V_{P}$ for the QPC, consistent with the onset of the conductance trace (Fig. 1). Therefore, from the peak $\mathrm{C}$ positions, we can determine the electron densities in the QPC channel using: $n_{Q P C}=f_{C} \times e / h$.
Assuming that only one valley is present in the channel, there are two possible cyclotron orbit orientations and the width $w$ can be calculated for each case based on Eqs. 13 as shown in Fig. [6. Using a scanning electron microscope, we confirmed that the QPC geometrical width is indeed $300 \mathrm{~nm}$, consistent with the width intended in our electron-beam lithography process. This implies that the width deduced for $X$ valley is not realistic since it exceeds $300 \mathrm{~nm}$ at high gate voltage. We conclude that it is the $Y$ valley that is occupied in the QPC and therefore dominates the transport across the QPC channel. This gives channel widths ranging from 50 to $130 \mathrm{~nm}$ (Fig. 6), implying depletion widths of 85 to $125 \mathrm{~nm}$ on each side of the channel wall. These are reasonable values for depletion widths in this type of structure ${ }^{21}$

\section{Quantum point contact model - revisited}

Having established that the QPC width increases with increasing gate voltage, we can now refine our QPC model (Fig. 17). As mentioned in Section II, the monotonic increase in plateau strength of the conductance trace with increasing $V_{G}$ suggests that the transport in the QPC arises from a single valley. This situation requires a residual valley splitting that further lifts the valley degeneracy and depopulates one of the valleys. Indeed from the Fourier spectrum of $d R_{x y} / d B$ in Fig. 國(b), where the spectrum represents $\mathrm{SdH}$ oscillation components arising from the 2D reservoir only, the half valley density peak $\mathrm{D}$ (at $\sim 2.5 \mathrm{~T}$ ) and half total density peak $\mathrm{B}($ at $\sim 7 \mathrm{~T})$ indicate a valley population imbalance. This imbalance corresponds to a valley splitting of $\Delta E_{V} \simeq 0.5 \mathrm{meV}$. In the presence of such a residual valley splitting, the Fermi energy of the majority valley is given as $E_{F}=n_{2 D} / \rho+\Delta E_{V} / 2$, as plotted in Fig. U(c).

The presence of a residual valley splitting in our sample is not unusual. It normally arises from a built-in residual strain introduced during sample cooldown. Furthermore, this valley splitting could be enhanced in the QPC channel. First, it is possible that additional anisotropic strain may be introduced during the fabrication process steps such as etching and gate evaporation. Second, the additional confinement and lower electron density in the QPC region could lead to stronger exchange interaction and make the system more easily valley polarized ${ }^{28}$ Thus it is not unlikely that the transport in the QPC channel is valley polarized.

Therefore, in the refined model, we assume that there is only one valley that dominates the transport in the QPC, in this case the $Y$ valley as suggested from the previous section and Fig. [6. Using an infinite square well model with channel width $w$ as an adjustable parameter, we can calculate the $1 \mathrm{D}$ energy subbands to fit the expected level crossings as shown in Fig. 7(c). We assume the width $w$ is a monotonic and smooth function of the gate voltage. As we increase $E_{F}$, the QPC channel gets wider and the quantized energy levels drop. We 


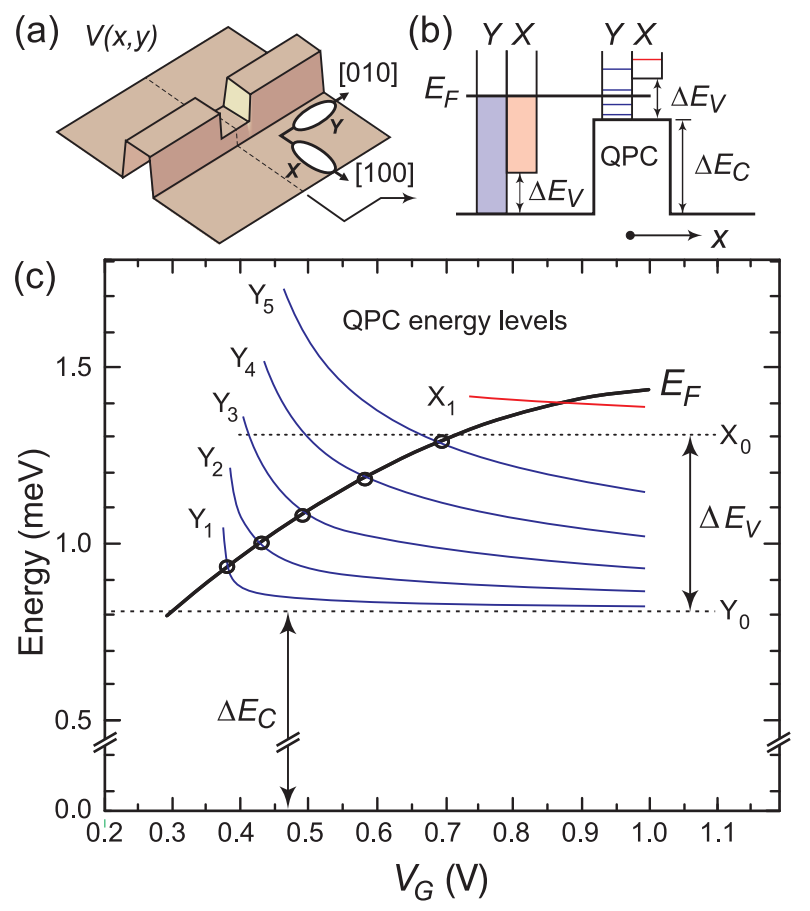

FIG. 7: (a) Potential landscape surrounding the QPC. (b) Potential cross-section along the transport direction. A finite residual valley splitting $\left(\Delta E_{V}\right)$ in the $2 \mathrm{D}$ reservoir is shown. In the QPC this valley splitting is enough to completely depopulate the $X$ valley. (c) A revised QPC energy level model with variable channel width. The model fits the QPC energy levels to all observed level crossings.

obtain the gate voltage-dependent width $w\left(V_{G}\right)$ as the fitting parameter and plot $w$ as a solid curve in Fig. 6 for comparison. We find that the widths deduced from the QPC model fall fairly close, within the error band, to the widths deduced from the negative MR kink. This self consistency indicates that indeed it is the $Y$ valley that dominates the transport in the QPC, while the $X$ valley in the QPC is depopulated due to a residual valley splitting.

The resulting energy level spacings between successive quantized energy levels are $\sim 0.1 \mathrm{meV}$, very small compared to typical subband spacing of most GaAs QPC devices ( $\sim 5$ to $20 \mathrm{meV}$ ), yet still somewhat larger than the thermal energy $\sim 26 \mu \mathrm{eV}$ at $T=0.3 \mathrm{~K}$. This is consistent with the fact that we observe only rather weak developing, quantized conductance plateaus.

We would like to point out that we can interpret the data of Fig. [7with the $X$ valley instead of $Y$ as the source of the quantized energy levels, and $m_{t}$ as the relevant effective mass along the confinement potential. In order to yield the same energy levels, such an interpretation requires a larger channel width, whose values fall near (slightly above) curve $X$ in Fig. 6] This happens because the quantized energy levels $E_{N}$ scale as $1 / m^{*} w^{2}$ and, to obtain the same energy levels, the channel width has to be larger by $\sqrt{m_{l} / m_{t}}$. However, as mentioned in section

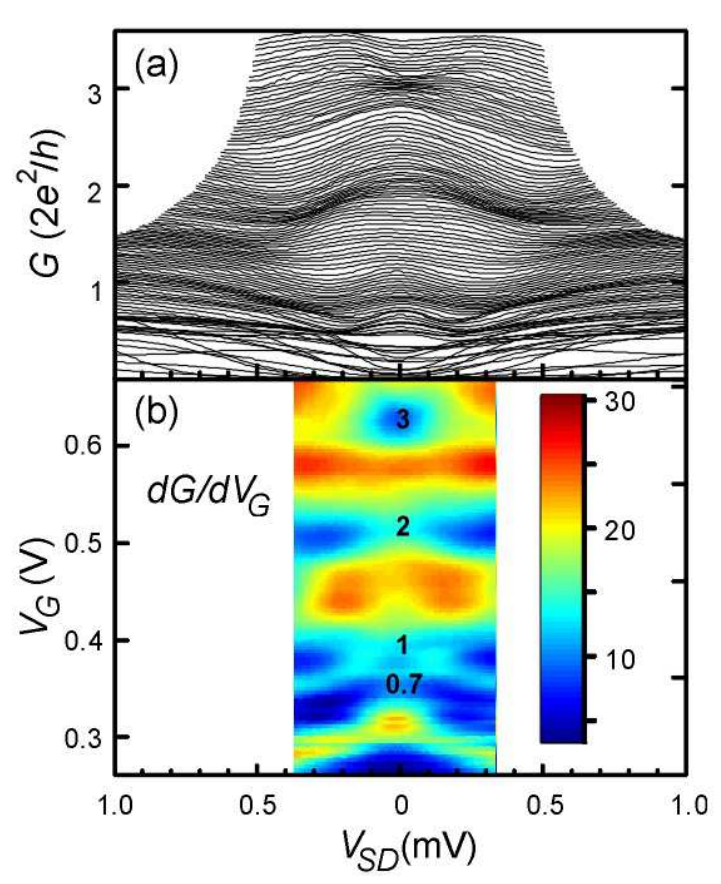

FIG. 8: (a) Differential conductance $G=d I / d V_{S D}$ traces measured at $T=0.3 \mathrm{~K}$. Each trace corresponds to incremental step of $2 \mathrm{mV}$ in $V_{G}$ starting from $0.3 \mathrm{~V}$ at the bottom. (b) The transconductance $\left(d G / d V_{G}\right)$ spectrum. The bluish (dark) regions correspond to developing plateaus. The numbers represent the plateaus indices.

IV.B, the widths deduced for the case of $X$ valley are not realistic as they exceed the physical channel width of 300 $\mathrm{nm}$ at high $V_{G}$.

\section{SOURCE DRAIN BIAS SPECTROSCOPY}

Having provided a basic understanding of the QPC conductance data vs $V_{G}$ in our system, we proceed to focus on the " 0.7 structure". A relevant measurement is the finite source-drain bias spectroscopy 5.12 .15 We measured the differential conductance $G=d I / d V_{S D}$ by applying a small AC excitation signal superposed on a DC source-drain bias $\left(V_{S D}\right)$. Figure 8 (a) shows that the measured source-drain bias conductance traces exhibit nonlinear transport behavior through the QPC. We observe a bunching of the traces along the $V_{S D}=0$ line that indicate the formation of plateaus at integer multiples of $2 e^{2} / h$.

These features in the conductance traces can be seen more clearly in the transconductance spectrum $d G / d V_{G}$ obtained numerically. This spectrum is displayed in Fig. \& (b) where the blue color indicates minima in $d G / d V_{G}$ that correspond to developing conductance plateaus at integer multiples of $2 e^{2} / h$. If the plateaus are well developed one can extract the energy subband spacings in the QPC $\stackrel{12.29}{ }$ Unfortunately, since the plateaus 


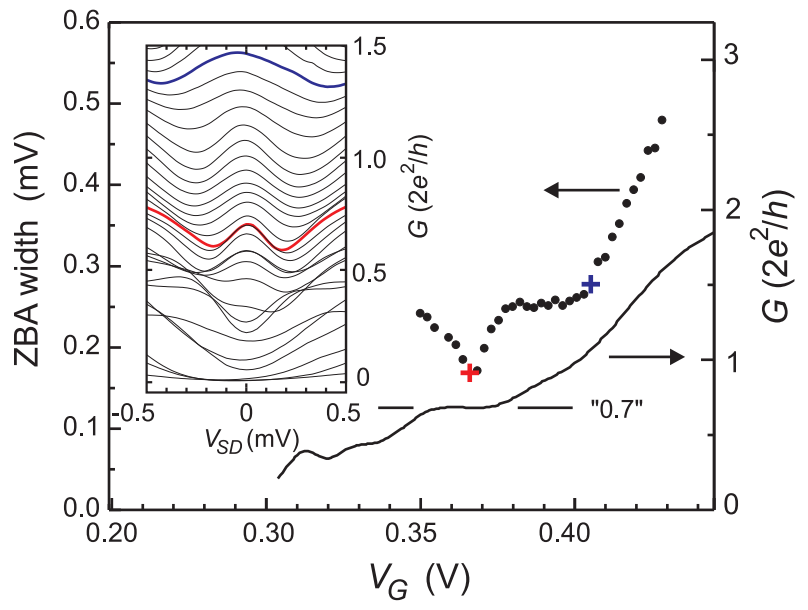

FIG. 9: Full-width at half-maximum (FWHM) of the ZBA peak as a function of gate voltage. The color data points (plus signs) are associated with the conductance trace of the same color in the inset. For reference the conductance trace $G$ vs $V_{G}$ is replotted. Inset: Differential conductance $G=d I / d V_{S D}$ at low conductance where the ZBA occurs.

are weak in our device, we cannot deduce the energy subband level spacings from this data.

However, one particularly interesting feature of the non-linear conductance measurements, relevant to the "0.7 structure", is the conductance peak around zero $V_{S D}$, known as the zero bias anomaly (ZBA) $\stackrel{15}{=}$ This feature can be observed more clearly in the expanded plot of the $V_{S D}$ bias spectrum as shown in the inset of Fig. 9 The ZBA below the first $2 e^{2} / h$ plateau has been observed previously in other systems such as in GaAs ${ }^{15}$ and GaN ${ }^{5}$ QPCs and has been associated with a Kondo-like correlated state. The width of the ZBA was found to show a dip near the " 0.7 structure" and increase monotonically with increasing gate voltage $e^{5.15}$ Here, we observe qualitatively similar behavior (Fig. 9). It was argued in Ref. 15 that the widths of the ZBA peaks, after the dip, are close to $2 k_{B} T_{K} / e$ where $T_{K}$ is the Kondo temperature of the system. However, we remark on a difference here. It appears in our data that, for our device, the ZBA persists above the first quantized plateau $\left(2 e^{2} / h\right)$ and its width keeps increasing, while in the GaAs system the ZBA width diverges as the conductance traces collapse to the first plateau $\stackrel{15}{15}$ This could simply be related to the fact that our QPC does not show a strong plateau at $2 e^{2} / h$.

\section{SUMMARY AND CONCLUSIONS}

We have studied transport in a QPC device fabricated in a two-dimensional electron system confined to an 11 nm-wide AlAs QW. The QPC is defined by shallowetched regions and entirely covered by a top gate that controls the density in both the $2 \mathrm{D}$ reservoirs and the QPC channel. The conductance trace obtained shows developing quantized conductance plateaus at integer steps of $2 e^{2} / h$. From the density measurement in the $2 \mathrm{D}$ reservoirs and in the QPC channel we construct a simple model with a gate-voltage dependent channel width to describe the crossings of the Fermi energy and the QPC quantized energy levels. The QPC model and the channel width determination using the negative magnetoresistance kink corroboratively indicate that the transport in the QPC channel is dominated by the $Y$ valley, the valley with higher mass along the QPC lateral confinement direction. This is a plausible outcome since the lowest QPC subband energy levels should be dominated by the carriers with higher effective mass along the confinement direction. Our results highlight the intricacies of understanding quantized conductance in a system with multiple and anisotropic valleys such as ours. Additionally, we observe a well-developed " 0.7 structure" that may reflect strong electron-electron interaction in this system thanks to its heavier electron mass.

We close by making a few remarks. There has been considerable interest recently in utilizing the spin degree of freedom to make functional devices (spintronics) 30 or to serve as a qubit for quantum computation ${ }^{31}$ The valley degree of freedom may provide an alternative for such applications $\stackrel{28}{2}$ The QPC can then be utilized as a simple valley filter, a potentially important device component, where the QPC confinement breaks the valley degeneracy, favoring the carriers in one valley and thus leading to valley polarized transport. In contrast, a spin filter is much more difficult to implement ${ }^{32}$ Furthermore, by simply orienting the QPC transport directions along either [100] or [010], one can choose which valley species to filter. Alternatively, one could preserve the valley degeneracy in the QPC by orienting the QPC with its transport direction along [110]. Finally, it is possible to control the valley populations by applying tunable strain using a piezo-actuator to which the sample is glued $3^{33}$

\section{ACKNOWLEDGEMENT}

We thank the NSF and ARO for support, and K. Vakili, Y. P. Shkolnikov, D. Goldhaber-Gordon and J. Shabani for illuminating discussions.
* Current address: Department of Physics, Columbia university, New York, NY 10027
1 B. J. van Wees, H. van Houten, C. W. J. Beenakker, J. G. 
Williamson, L. P. Kouwenhoven, D. van der Marel, and C. T. Foxon, Phys. Rev. Lett. 60, 848 (1988).

2 D. A. Wharam, T. J. Thornton, R. Newbury, M. Pepper, H. Ahmed, J. E. F. Frost, D. G. Hasko, D. C. Peacock, D. A. Ritchie, and G. A. C. Jones, J. Phys. C. 21, L209 (1988).

3 D. Tobben, D. A. Wharam, G. Abstreiter, J. P. Kotthaus, and F. Schaffler, Semicond. Sci. Technol. 10, 711 (1995).

${ }^{4}$ U. Wieser, U. Kunze, K. Ismail, and J. O. Chu, Appl. Phys. Lett. 81, 1726 (2002).

${ }^{5}$ H. T. Chou, S. Lüscher, D. Goldhaber-Gordon, M. J. Manfra, A. M. Sergent, K. W. West, and R. J. Molnar, Appl. Phys. Lett. 86, 73108 (2005).

6 N. Goel, J. Graham, J. C. Keay, K. Suzuki, S. Miyashita, M. B. Santos, and Y. Hirayama, Physica E 26, 455 (2005).

7 L. P. Rokhinson, L. N. Pfeiffer, and K. W. West, Phys. Rev. Lett. 96, 156602 (2006).

8 R. Danneau, W. R. Clarke, O. Klochan, A. P. Micolich, A. R. Hamilton, M. Y. Simmons, M. Pepper, and D. A. Ritchie, Appl. Phys. Lett. 88, 12107 (2006).

9 T. P. Smith III et al., Phys. Rev. B 35, 9349 (1987); H. W. van Kesteren et al., Phys. Rev. B 39, 13426 (1989); K. Maezawa et al., J. Appl. Phys. 71, 296 (1992); T. S. Lay et al., Appl. Phys. Lett. 62, 3120 (1993); S. Yamada et al., Physica B 201, 295 (1994).

10 Small discrepancies between the actual plateau positions and $N \times 2 e^{2} / h$ levels are present due to background resistances of 100 to $800 \Omega$, which are typical values for resistance of the $2 \mathrm{D}$ reservoir regions.

11 A. Kristensen, J. B. Jensen, M. Zaffalon, C. B. Sørensen, S. M. Reimann, P. E. Lindelof, M. Michel, and A. Forchel, J. Appl. Phys. 83, 607 (1998).

12 A. Kristensen, H. Bruus, A. E. Hansen, J. B. Jensen, P. E. Lindelof, C. J. Marckmann, J. Nygård, C. B. Sørensen, F. Beuscher, A. Forchel, and M. Michel, Phys. Rev. B 62, 10950 (2000).

13 E. P. De Poortere, Y. P. Shkolnikov, E. Tutuc, S. J. Papadakis, M. Shayegan, E. Palm, and T. Murphy, Appl. Phys. Lett. 80, 1583 (2002).

14 K. J. Thomas, J. T. Nicholls, M. Y. Simmons, M. Pepper, D. R. Mace, and D. A. Ritchie, Phys. Rev. Lett. 77, 135 (1996).

15 S. M. Cronenwett, H. J. Lynch, D. Goldhaber-Gordon, L. P. Kouwenhoven, C. M. Marcus, K. Hirose, N. S. Wingreen, and V. Umansky, Phys. Rev. Lett. 88, 226805 (2002).

16 D. J. Reilly, T. M. Buehler, J. L. O‘Brien, A. R. Hamilton,
A. S. Dzurak, R. G. Clark, B. E. Kane, L. N. Pfeiffer, and K. W. West, Phys. Rev. Lett. 89, 246801 (2002).

17 A. C. Graham, K. J. Thomas, M. Pepper, N. R. Cooper, M. Y. Simmons, and D. A. Ritchie, Phys. Rev. Lett. 91, 136404 (2003).

18 P. Roche, J. Ségala, D. C. Glattli, J. T. Nicholls, M. Pepper, A. C. Graham, K. J. Thomas, M. Y. Simmons, and D. A. Ritchie, Phys. Rev. Lett. 93, 116602 (2004).

19 J. Moser, T. Zibold, D. Schuh, M. Bichler, F. Ertl, G. Abstreiter, M. Grayson, S. Roddaro, and V. Pellegrini, Appl. Phys. Lett. 87, 52101 (2005).

20 E. P. De Poortere, Y. P. Shkolnikov, and M. Shayegan, Phys. Rev. B 67, 153303 (2003).

21 K. K. Choi, D. C. Tsui, and K. Alavi, Appl. Phys. Lett. 50, 110 (1987).

22 Y. Takagaki, T. Kosugi, K. Gamo, S. Namba, and K. Murase, Semicond. Sci. Technol. 5, 634 (1990).

${ }^{23}$ H. van Houten, C. W. J. Beenakker, P. H. M. van Loosdrecht, T. J. Thornton, H. Ahmed, M. Pepper, C. T. Foxon, and J. J. Harris, Phys. Rev. B 37, 8534 (1988).

24 O. Gunawan, Y. P. Shkolnikov, E. P. De Poortere, E. Tutuc, and M. Shayegan, Phys. Rev. Lett. 93, 246603 (2004).

${ }^{25}$ K. F. Berggren, T. J. Thornton, D. J. Newson, and M. Pepper, Phys. Rev. Lett. 57, 1769 (1986).

26 W. Pan, J. S. Xia, H. L. Stormer, D. C. Tsui, C. L. Vicente, E. D. Adams, N. S. Sullivan, L. N. Pfeiffer, K. W. Baldwin, and K. W. West, Phys. Rev. Lett. 95, 66808 (2005).

27 Y. P. Shkolnikov, E. P. De Poortere, E. Tutuc, and M. Shayegan, Phys. Rev. Lett. 89, 226805 (2002).

28 O. Gunawan, Y. P. Shkolnikov, K. Vakili, T. Gokmen, E. P. De Poortere, and M. Shayegan, cond-mat/0605692.

29 N. K. Patel, J. T. Nicholls, L. Martin-Moreno, M. Pepper, J. E. F. Frost, D. A. Ritchie, and G. A. C. Jones, Phys. Rev. B 44, 13549 (1991).

30 S. A. Wolf, D. D. Awschalom, R. A. Buhrman, J. M. Daughton, S. von Molnar, M. L. Roukes, A. Y. Chtchelkanova, and D. M. Treger, Science 294, 1488 (2001).

31 J. R. Petta, A. C. Johnson, J. M. Taylor, E. A. Laird, A. Yacoby, M. D. Lukin, C. M. Marcus, M. P. Hanson, and A. C. Gossard, Science 309, 2180 (2005).

32 J. A. Folk, R. M. Potok, C. M. Marcus, and V. Umansky, Science 299, 679 (2003).

33 M. Shayegan, K. Karrai, Y. P. Shkolnikov, K. Vakili, E. P. De Poortere, and S. Manus, Appl. Phys. Lett. 83, 5235 (2003). 